\title{
Venous thromboembolism in childhood
}

\author{
Kalyani Guruge $^{1}$ \\ Sri Lanka Journal of Child Health, 2009; 38: 28-33
}

(Key words: venous thromboembolism, VTE, childhood)

\section{Introduction}

There is growing awareness that venous thromboembolism (VTE) occurs in the paediatric age group and it is recognized as a critical paediatric concern ${ }^{1}$. The survival of children from serious underlying primary diseases such as congenital heart disease or malignancy and the use of central venous catheters have significantly contributed to the increased incidence of VTE. Additionally, some prothrombotic disorders are thought to contribute to the presentation.

There are numerous clinical classifications of VTE. The use of precise description assists in optimizing clinical care ${ }^{1}$. Clinically VTE is described as first episode versus recurrent, symptomatic versus asymptomatic, acute versus chronic and idiopathic versus risk associated disease ${ }^{1}$. Anatomical descriptions include lower extremity versus upper extremity, distal versus proximal, deep versus superficial and peripheral versus central (cerebral and pulmonary) ${ }^{1}$.

\section{Epidemiology}

The incidence of VTE varies widely depending on the study design and diagnostic tests used to detect thrombosis. The incidence is remarkably lower in children compared to adults. The National Hospital Discharge survey in the United States of America (USA) (1979-2001) has disclosed an incidence of strokes approximately 4.9 cases per 100,000 children per year ${ }^{2}$.

The age distribution of the incidence rate is bimodal with peak rates in the neonatal period and adolescence $^{1}$. The Dutch registry, for instance, has indicated a VTE incidence rate of 14.5 per 100,000 in the neonatal period compared with an overall incidence rate of 1.4 per 100,000 children per year ${ }^{3}$.

${ }^{1}$ Consultant Paediatrician, Lady Ridgeway Hospital, Colombo.

Further, the VTE-specific incidence rate in USA among adolescents 15 to 17 years of age was 11 per 100,000 per year, a rate nearly threefold that observed overall in children ${ }^{2}$. In teenage years the incidence was greater in girls, pregnancy and oral contraceptive pills probably contributing to the difference $^{2}$.

In the analysis of the Canadian registry data the documented VTE in upper and lower venous system was 5.3 per 1000 hospital admissions with a mortality of $2.2 \%{ }^{4}$. The mortality rate was predominantly due to associated conditions, which were identified in $96 \%$ of children; a central (indwelling) venous line was the single most important predisposing factor for VTE, being present in $33 \%$ of cases ${ }^{4}$.

\section{Pathophysiology}

The pathogenesis of VTE is explained by Virchow $\operatorname{triad}^{1}$ as shown in figure 1 .

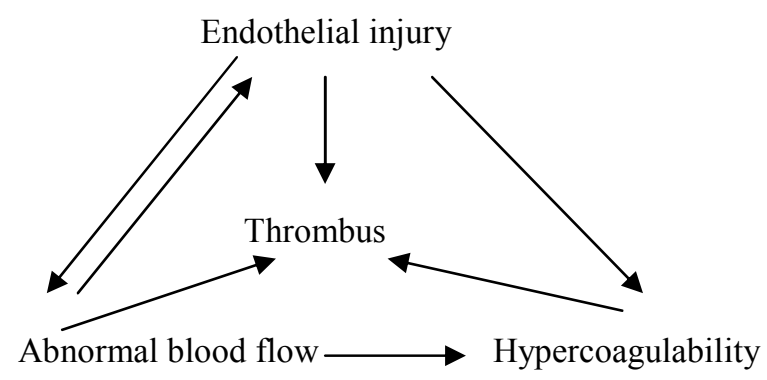

Figure 1 - Virchow triad

\section{Clinical prothrombotic risk factors}

Some clinical prothrombotic risk factors ${ }^{1}$ are shown in table 1 . 
Table I

Clinical prothrombotic risk factors

\begin{tabular}{|l|l|l|}
\hline \multicolumn{1}{|c|}{$\begin{array}{c}\text { Endothelial } \\
\text { injury }\end{array}$} & \multicolumn{1}{|c|}{$\begin{array}{c}\text { Abnormal } \\
\text { blood flow }\end{array}$} & $\begin{array}{c}\text { Hypercoagulable } \\
\text { state }\end{array}$ \\
\hline $\begin{array}{l}\text { Indwelling } \\
\text { venous catheter }\end{array}$ & Immobilization & $\begin{array}{l}\text { Inherited } \\
\text { thrombophilia }\end{array}$ \\
\hline Sepsis & Sedentary state & $\begin{array}{l}\text { Acquired } \\
\text { thrombophilia }\end{array}$ \\
\hline Trauma & $\begin{array}{l}\text { Post-operative } \\
\text { state }\end{array}$ & \\
\hline Chemotherapy & Dehydration & \\
\hline $\begin{array}{l}\text { Anti } \\
\text { phospholipid } \\
\text { antibodies }\end{array}$ & Inflammation & \\
\hline & Hyperviscosity & \\
\hline & $\begin{array}{l}\text { Nephrotic } \\
\text { syndrome }\end{array}$ & \\
\hline & Cancer therapy & \\
\hline
\end{tabular}

In children, more than $90 \%$ of VTE is associated with more than one component of the triad ${ }^{3,5}$ e.g. presence of an indwelling catheter in a child with infectious illness and underlying malignancy or catheter in a child with congenital heart disease and corrective surgery.

Indwelling venous catheter is the most significant risk factor for development of VTE. More than 50\% of cases of deep vein thrombosis (DVT) in children and more than $80 \%$ of cases in neonates occur in association with central venous lines ${ }^{4,6}$.

Inherited and acquired thrombophilic conditions constitute the third component of the Virchow triad ${ }^{1}$. Thrombophilia in children is most often acquired but rarely may be congenital (anticoagulant deficiency) ${ }^{1}$. Thrombophilia is explained by any alteration in the haemostatic balance that increases thrombin production, enhances platelet activation or aggregation, mediates endothelial activation or damage or inhibits fibrinolysis ${ }^{1}$. Increased factor VIII activity with significant infection and inflammatory states, anticoagulant deficiencies resulting from consumption in bacterial sepsis and disseminated intravascular coagulation (DIC) or production of inhibitory antibodies in acute viral infection and parainfectious development of anti phospholipid antibodies are examples of acquired thrombophilia in children ${ }^{1}$.

\section{Clinical Presentation}

Clinical suspicion for acute VTE should be high in the following circumstances ${ }^{1}$ :

1. Presence of prothrombotic risk factors.
2. Family history of early VTE or other vascular disease.

3. Known thrombophilia traits.

4. Clinical signs and symptoms.

\section{Signs and symptoms of deep vein thrombosis} $(\boldsymbol{D V} T)^{1}$

1. Painful, unilateral limb swelling (Homan sign, palpable cord in the popliteal fossa, may be absent).

2. Warm and plethoric extremity.

3. Poorly functioning venous cannula.

4. Swelling of neck and face, bilateral periorbital oedema and headache (DVT in the upper limb, with extension to the superior vena cava (SVC)).

Signs and symptoms of pulmonary embolism $(P E)^{1}$

1. Unexplained shortness of breath of sudden onset.

2. Pleuritic chest pain.

3. Haemoptysis.

4. Tachycardia.

5. Hypoxaemia (if PE is proximal or extensively bilateral).

6. Cyanosis and sudden collapse.

7. Right heart failure with hepatomegaly or peripheral oedema.

8. May be asymptomatic or produce few symptoms ${ }^{7}$.

Signs and symptoms of acute cerebral sinovenous thrombosis (CSVT) ${ }^{1}$

1. Unusually severe or persistent headache.

2. Blurring of vision.

3. Cranial nerve palsy.

4. Papilloedema.

5. Seizures.

Signs and symptoms of renal vein thrombosis $(R V T)^{1}$

1. Haematuria and thrombocytopenia.

3. Oliguria and uraemia if bilateral.

4. Flank mass especially in neonates.

5. Peripheral and periorbital oedema in older children often with nephrotic syndrome

\section{Signs and symptoms of intracardiac thrombosis ${ }^{1}$}

1. Catheter - related or post cardiac surgery thrombosis is often asymptomatic.

\section{Signs and symptoms of portal vein thrombosis ${ }^{1}$}

1. Splenomegaly

2. Anaemia 
3. Gastrointestinal bleeding due to gastrooesophageal varices.

\section{Signs and symptoms of chronic VTE}

1. May be diagnosed incidentally without signs or symptoms.

2. May present with signs and symptoms of chronic venous obstruction or post thrombotic syndrome (PTS):
a. Limb pain and oedema
b. Dilated superficial collateral veins
c. Venous stasis dermatitis
d. Ulceration of the skin

\section{Diagnosis}

Venography has been the gold standard for diagnosis but is limited by being an invasive investigation ${ }^{1}$. Presently there are 'non-invasive' or 'minimally invasive but effective' radiological imaging techniques which confirm the diagnosis and help in defining the extent and occlusiveness of the thrombus ${ }^{1}$.

\section{Radiological imaging ${ }^{1}$}

1. Distal or proximal lower extremity - compression ultrasonography with Doppler imaging.

2. Deep pelvic or abdominal veins - CT or MRI.

3. Upper limb - ultrasound with Doppler can yield false negative results as clavicle hinders the view of jugular and axillary veins.

4. SVC or right atrium - Echocardiography, CT or MRI.

5. Jugular venous system (suspected Lemierre syndrome) - compression ultrasonography with Doppler imaging.

6. Pulmonary embolism - spiral CT. Ventilationperfusion scan is of limited availability and expertise with this modality is limited).

7. CSVT - CT or CT venography, MRI or MR venography.

8. RVT - Doppler ultrasound (neonates) Doppler ultrasound or CT (older children)

9. Portal vein thrombosis - Doppler ultrasound or CT.

MR venography is more expensive than CT venography and requires sedation in children less than 8 years of age or those who are developmentally delayed or very anxious ${ }^{1}$. However, the diagnostic sensitivity of MR venography is as good as CT without the danger of significant radiation ${ }^{1}$.

\section{Laboratory investigations}

Once a clot is demonstrated the patient's workup should include:

1. Full blood count with peripheral blood picture. It is important to document a normal platelet count before heparin is started.

2. Prothrombin time (PT), activated partial thromboplastin time (aPTT) and fibrinogen level. Prolonged PT + aPTT and/or low fibrinogen level suggest disseminated intravascular coagulation (DIC).

Prolonged aPTT indicates lupus anticoagulant.

3. D-dimer measurement - paediatric studies to assess the usefulness are limited. Children often have other disorders such as sepsis or malignancy which may increase the D-dimer concentration.

\section{Workup for hypercoagulation}

1. Protein $\mathrm{C}$ functional assay and activated protein $\mathrm{C}$ resistance.

2. Protein $\mathrm{S}$ antigen - free and total

3. Factor V Leiden mutation

4. Anti thrombin assay

5. Lupus anticoagulant

6. Anti cardiolipin antibodies

7. Prothrombin gene 20210A mutation

8. Lipoprotein levels

9. Plasma homocystine value

Heparin therapy affects antithrombin, protein $\mathrm{C}$ and $\mathrm{S}$ and activated protein $\mathrm{C}$ resistance. Warfarin affects protein $\mathrm{C}, \mathrm{S}$ and antithrombin. Both drugs have no effect on anticardiolipin antibodies, factor $\mathrm{V}$ Leiden mutation, prothrombin gene mutation or lipoprotein level.

\section{Treatment}

\section{Therapeutic goals}

1. Prevent thrombus propagation and/or embolization.

2. Restore blood flow (rapidly if possible).

3. Minimize long term sequelae.

Initial care and evaluation occur in the paediatric ward but if severe respiratory distress or neurological deterioration is present, patient management should be in intensive care setup. 


\section{Surgical care}

Thrombectomy is necessary after major cardiac surgery or if thrombolytic agents fail or are contraindicated. Paediatric haematologist, vascular surgeon, and when necessary, neurologist and oncologist should be involved with the paediatrician or neonatologist in providing care.

\section{Diet}

Vitamin $\mathrm{K}$ directly interferes with effectiveness of warfarin and may increase the risk for re-thrombosis. Ingestion of vitamin $\mathrm{K}$ rich green leafy vegetables and maternal intake of vitamin $\mathrm{K}$ in breast fed babies should be kept at a minimal level. Vitamin $\mathrm{K}$ in parenteral nutrition should be removed.

\section{Activity}

Children are given bed rest for the first 24-48 hours to decrease the risk of pulmonary embolism. Children with lower extremity DVT are fitted with compression stockings.

\section{Anticoagulant treatment}

Heparin and warfarin are the most commonly used anticoagulants in children ${ }^{1}$. Heparin includes unfractionated heparin (UFH) and low molecular weight heparin $(\mathrm{LMWH})^{1}$. Heparin enhances the activity of antithrombin, an intrinsic anticoagulant protein that serves as a key inhibitor of thrombin ${ }^{1}$. Warfarin acts through antagonism of vitamin $\mathrm{K}$, thereby interfering with gamma-carboxylation of the vitamin K-dependent procoagulant factors II, VII, IX and $\mathrm{X}$ and intrinsic anticoagulant proteins $\mathrm{C}$ and $\mathrm{S}^{1}$. UFH is given intravenously whilst LMWH is given subcutaneously and vitamin $\mathrm{K}$ is given orally ${ }^{1}$. LMWH has a decreased risk for the development of thrombocytopenia compared with $\mathrm{UFH}^{1}$. UFH has a shorter half-life than LMWH and is preferred in circumstances of heightened bleeding risk or labile acute clinical status ${ }^{1}$. Heparin can be reversed with protamine and warfarin with vitamin $\mathrm{K}$. The lab must be made aware of the type of heparin used so that appropriate assay standard is used. Warfarin therapy is started after therapeutic anticoagulation is achieved with heparin.

Recommended antithrombotic therapy for first and recurrent episodes according to the seventh ACCP guidelines ${ }^{8}$ is shown in table 2

Table 2

Recommended treatment and duration

\begin{tabular}{|c|l|l|l|}
\hline Episode & \multicolumn{1}{|c|}{ Initial therapy } & \multicolumn{1}{c|}{ Extended therapy } & \multicolumn{1}{c|}{ Duration } \\
\hline First & UFH 0.3-0.7 U/ml & $\begin{array}{l}\text { Warfarin }\left(2^{\text {nd }} \text { or } 3^{\text {rd }} \text { day) }\right. \\
\text { Keep INR } 2.0-3.0 \text { (checked } \\
\text { after 5 days of initiation and } \\
\text { weekly thereafter until stable) } \\
\text { LMWH 0.5-1.0 U/ml }\end{array}$ & $\begin{array}{l}\text { Resolved or transient risk factor: 3-6 } \\
\text { months } \\
\text { Idiopathic or no known risk factor: 6-12 } \\
\text { months } \\
\text { Chronic clinical risk factor: 6-12 months } \\
\text { Potent congenital thrombophilia: indefinite }\end{array}$ \\
\hline Recurrent & UFH 0.3-0.7 U/ml & $\begin{array}{l}\text { Warfarin }\left(2^{\text {nd }} \text { or } 3^{\text {rd }} \text { day) }\right. \\
\text { Keep INR 2.0-3.0 (checked } \\
\text { after 5 days of initiation and } \\
\text { Leekly thereafter until stable) } \\
\text { LMWH 0.5-1.0 U/ml }\end{array}$ & $\begin{array}{l}\text { Resolved risk factor: 6-12 months } \\
\text { Idiopathic 12 months } \\
\text { Chronic clinical risk factor: indefinite } \\
\text { Potent congenital thrombophilia: indefinite }\end{array}$ \\
\hline
\end{tabular}

Anti thrombotic therapy with anticoagulants has been used for many years but thrombolytic therapy is now gaining recognition especially in children with significant PE or extensive limb-threatening VTE ${ }^{1}$. These drugs promote fibrinolysis whereas anticoagulants attenuate hypercoagulability ${ }^{1}$. Tissuetype plasminogen activator is administered as a recombinant agent by different routes (e.g. systemic bolus, short-duration infusion, low-dose continuous infusion, local catheter-directed infusion with or without mechanical thrombectomy $)^{1}$. Thrombolysis regimen followed by standard anticoagulation may reduce the risk for post thrombotic syndrome substantially compared with standard anticoagulation alone ${ }^{9}$.

Other antithrombotic agents include factor $\mathrm{Xa}$ inhibitors and thrombin inhibitors ${ }^{1}$. Hirudin is a direct thrombin inhibitor ${ }^{1}$. Protein $\mathrm{C}$ concentrate is still undergoing clinical trials. It may be useful in the treatment of purpura fulminans resulting from microvascular thrombosis in children with sepsis 
especially meningococcaemia ${ }^{10}$. Antithrombin replacement combined with daily prophylactic LMWH during induction and consolidation phases of therapy in acute lymphoblastic leukaemia (ALL) has a potential benefit for VTE risk reduction ${ }^{11}$. Antithrombin also has a role to play in nephrotic syndrome associated VTE (antithrombin deficiency prevents achievement of therapeutic anti-Xa levels i.e. heparin resistance) and in neonates with clinical conditions when antithrombin consumption is high ${ }^{1}$.

The use of vena caval filters should be considered in children of appropriate size in whom recurrent VTE (especially PE) occurs on therapeutic anticoagulation in the presence of a persistent prothrombotic risk factor $^{1}$. In addition, temporary vena caval filters may be considered during times of especially heightened risk of $\mathrm{PE}^{1}$. With regard to long-standing vena caval filters, the impact of such non-retrievable devices on the vena cava of developing children has not been studied ${ }^{1}$.

\section{Outcomes}

\section{Short term adverse outcomes of $\mathrm{VTE}^{1}$}

1. Major haemorrhagic complications of antithrombotic interventions.

2. Major haemorrhagic complications of the thrombotic event itself e.g. post thrombotic haemorrhage in the brain, adrenal gland or testis.

3. Early recurrent VTE (including DVT and PE).

4. SVC syndrome in DVT of the upper venous system.

5. Acute renal insufficiency in RVT.

6. Pulmonary embolism.

7. Catheter related sepsis and catheter malfunction (sometimes necessitating surgical replacement).

8. Severe acute venous insufficiency leading to venous infarction with gangrene of the limbs in rare cases of occlusive DVT involving the extremities.

9. Death from haemodynamic instability in extensive intracardiac thrombosis or proximal PE

\section{Long term adverse outcomes of $V T E^{12}$}

1. Recurrent VTE.

2. Chronic hypertension and renal insufficiency in RVT.

3. Variceal haemorrhage in portal vein thrombosis.

4. Chronic SVC syndrome in catheter-related thromboembolism (CRT) involving SVC occlusion.

5. Loss of availability for venous access in recurrent or extensive CRT of the upper venous system.
6. Development of the post thrombotic syndrome (PTS), a condition of chronic venous insufficiency after DVT.

Children seem to have a lower risk for recurrent thromboembolism than adults ${ }^{1}$. The risk for PTS in children who have DVT of the limbs, however, is as great as that in adults ${ }^{1}$. VTE specific mortality in children is low, ranging from $0 \%$ to $2 \%{ }^{13}$. A considerably higher all-cause mortality reflects the severity of underlying conditions (e.g. sepsis, cancer and congenital cardiac disease) in paediatric $\mathrm{VTE}^{1}$. Neonate-specific outcomes data in paediatric nonRVT VTE reflect an all-cause mortality of $12 \%$ to $18 \%^{14}$

Outcomes of VTE in children differ with the anatomic site involved. In a Canadian study of CRT in children $^{15}$, VTE-specific mortality was $4 \%$ among all children and $20 \%$ among those children in whom CRT was complicated by $\mathrm{PE}^{15}$.

Persistence of thrombosis after anticoagulation is recognised. This is associated with venous valvular insufficiency but does not increase the risk of recurrent VTE or $\mathrm{PE}^{16}$. The prevalence of residual thrombosis despite adequate anticoagulation in neonatal VTE has ranged from $12 \%$ in a small series of premature newborns who had $\mathrm{CRT}^{17}$ to $62 \%$ in full-term neonatal VTE survivors ${ }^{4}$.

Prediction of long term outcome of VTE during acute phase helps in planning out the antithrombotic management ${ }^{1}$.

APA syndrome, homozygous anticoagulant deficiencies and presence of multiple thrombophilia traits are associated with recurrent $\mathrm{VTE}^{18}$. The radiologic finding of complete veno-occlusion at diagnosis of DVT is associated with an increased risk for persistent thrombosis ${ }^{19}$. Plasma factor VIII activity greater than $150 \mathrm{U} / \mathrm{dl}$ and D-dimer concentration greater than $500 \mathrm{ng} / \mathrm{ml}$ at the time of diagnosis of VTE in children and after 3-6 months of standard anticoagulation are shown to predict a composite adverse thrombotic outcome, characterized by persistent thrombosis, recurrent VTE or the development of PTS $^{20}$.

VTE has become an important and critical problem in the paediatric age group. Paediatric recommendations are mainly derived from adult trials and therefore need further evaluation by studies undertaken in children. 


\section{References}

1. Goldenberg NA, Bernard TJ. Venous thromboembolism in children. Paediatric Clinics of North America 2008; 55: 305-22.

2. Stein PD, Kayali R, Olsen RE, et al. Incidence of venous thromboembolism in infants and children: data from the National Hospital Discharge survey. Journal of Paediatrics 2004; 145: 563-5.

3. Van Ommen CH, Heijboer H, Buller HR, et al. Venous thromboembolism in childhood: a prospective two-year registry in the Netherlands. Journal of Paediatrics 2001; 139: 676-81.

4. Andrew M, David M, Adams M, et al. Venous thromboembolic complications in children: first analyses of the Canadian Registry of VTE. Blood 1994; 83: 1251-7.

5. Monagle $\mathrm{P}$, Adams $\mathrm{M}$, Mahoney $\mathrm{M}$, et al. Outcome of paediatric thromboembolic disease: a report from the Canadian Childhood Thrombophilia Registry. Paediatric Research 2000; 47: 763-6.

6. Schmidt B, Andrew M. Neonatal thrombosis: report of a prospective Canadian and international registry. Paediatrics 1995; 96: 939-43.

7. Van Ommen $\mathrm{CH}$, Peters M. Acute pulmonary embolism in childhood. Thrombosis Research 2006; 118 (1): 13-25.

8. Monagle $\mathrm{P}$, Chan A, Massicotte $\mathrm{P}$, et al. Antithrombotic therapy in children: the Seventh ACCP Conference on Antithrombotic and thrombotic therapy. Chest 2004; 126 (Suppl 3): 645S-87S.

9. Goldenberg NA, Knapp-Clevenger R, Durham JD, et al. A thrombolytic regimen for high risk deep vein thrombosis may substantially reduce he risk of postthrombotic syndrome in children. Blood 2007; 110: 45-53.

10. Rivard GE, David M, Farrell C, et al. Treatment of purpura fulminans in meningococcaemia with protein C concentrate. Journal of Paediatrics 1995; 126 (4): 646-52.
11. Meister B, Kropshofer G, Klein-Frank A, et al. Comparison of low-molecular-weight heparin and antithrombin versus antithrombin alone for the prevention of thrombosis in children with acute lymphoblastic leukaemia. Paediatric Blood Cancer 2008; 50 (2): 298-303.

12. Goldenberg NA. Long-term outcomes of venous thrombosis in children. Current Opinion in Haematology 2005; 12: 370-6.

13. Oren H, Devecioglu O, Ertem M, et al. Analysis of paediatric thrombotic patients in Turkey. Paediatric Haematology Oncology 2004; 21: 573-83.

14. NowakGottl U, Von Kries R, Gobel U. Neonatal symptomatic thromboembolism in Germany; 2 year survey. Archives of Disease in Childhood Fetal \& Neonatal Edition 1997; 76: F 163-7.

15. Massicotte MP, Dix D, Monagle P, et al. Central venous catheter related thrombosis in children: analysis of the Canadian Registry of Venous Thromboembolic complications. Journal of Paediatrics 1998; 133: 770-6.

16. Meissner MH, Manzo RA, Bergelin RO, et al. Deep venous insufficiency: the relationship between lysis and subsequent reflux. Journal of Vascular Surgery1993; 18: 596-605.

17. Michaels LA, Gurian M, Hagyi T, et al. Low molecular weight heparin in the treatment of venous and arterial thromboses in the premature infant. Paediatrics 2004; 114: 703-7.

18. Nowak-Gottl U, Junker R, Kruez W, et al. Risk of recurrent venous thrombosis in children with combined prothrombotic risk factors. Blood 2001; 97: 858-62.

19. Revel-Vilk S. Sharathkumar A, Massicotte P, et al. Natural history of arterial and venous thrombosis in children treated with low molecular weight heparin; longitudinal study by ultrasound. Journal of Thrombosis \& Haemostasis 2004; 2:42-6.

20. Goldenberg NA, Knapp-Clevenger R, MancoJohnson MJ, et al. Elevated plasma factor VIII and D-dimer levels as predictors of poor outcomes of thrombosis in children. New England Journal of Medicine 2004; 351: 1081-8. 